\title{
Turbulence and transport in two-dimensional magnetized electron plasmas
}

\author{
Dastgeer Shaikh* and P. K. Shuklat \\ Institute of Geophysics and Planetary Physics (IGPP), \\ University of California, \\ Riverside, CA 92521. USA. \\ and \\ Institut für Theoretische Physik IV and Centre for Plasma Science and Astrophysics, \\ Fakultät für Physik und Astronomie, \\ Ruhr-Universität Bochum, D-44780 Bochum, Germany
}

\begin{abstract}
Electron plasmas confined by an external magnetic field exhibit variations in a two-dimensional plane orthogonal to the confining magnetic field. A nonlinear fluid simulation code to investigate the properties of 2-D electron plasma wave turbulence in a nonuniform magnetoplasma has been developed. It is found that the presence of the density gradient convection by mean electric fields considerably influence the characteristic nonlinear interaction processes, such as the energy cascades and the cross-field electron transport. The initial random turbulent state evolves towards an intermittent state where forward cascade of vorticity coexists with an inverse cascade of electric potential fluctuations. The latter lead to the formation of large scale entities in 2D electron plasmas and can be alternatively understood by seeking exact nonlinear coherent vortex solutions in the form of a dipolar-like configuration. The energy cascades are governed typically by the Kolmogorov-like $k^{-5 / 3}$ spectrum. In agreement with the experimental observations, we find that the electron transport is improved significantly by the application of an externally imposed electric field.
\end{abstract}

*Electronic address: dastgeer@ucr.edu

†Electronic address: ps@tp4.rub.de 


\section{INTRODUCTION}

Electron plasma columns are routinely confined in experimental devices by means of an external magnetic field for hours. While such non-neutral plasmas [1] exhibit excellent stability property under ideal conditions, there exist critical issues that can potentially destabilize the electron plasma columns. For instance, the asymmetry of confining field and the presence of neutral gas particles are reported to often cause damages to the stability of the electron plasma columns [2]. During the process of rotation around the ambient field, the electron plasma particles collide with each other resulting thus in a cross-field electron transport. The cross field transport is therefore dominated by the electron-electron collisions in an electron plasma column, whereas collisions with the background neutrals do not contribute to the transport. However the advent of a rotating wall electric field imposed externally on the electron plasma column leads to an improvement in the stability [2]. An application of the externally applied radial electric field $\mathbf{E}$ in the presence of the confining orthogonal magnetic field $\mathbf{B}$ imparts a net poloidal drift $\mathbf{v}_{\perp} \approx\left(c / B^{2}\right) \mathbf{E} \times \mathbf{B}$ on the electron plasma column. Consequently, the plasma column rotates around the external magnetic field which supposedly enhances its stability.

There exist morphological correspondence between the guiding center flow of electrons in a strongly magnetized electron plasma and the two-dimensional (2D) incompressible Euler fluid [3]. In a uniform plasma, the electron fluid dynamics is governed by the Navier-Stokes equation (NSE), which basically describe the evolution of the electron vorticity. The NSE equation admits interesting electron fluid turbulence behavior and localized vortex motions in the form of clumps (positive vorticity) and holes (negative vorticity) [4]. The dynamics of electron plasma vortices in background vorticity distribution has been investigated both analytically and numerically [4] as well as experimentally [5, 6].

In a nonuniform electron magnetoplasma containing equilibrium electron density gradient and fixed ion background, the dynamics of 2D electron drift modes (EDMs) is governed by the modified NSE [7]. Possible stationary solutions of the latter can be cast in the form of a global vortex pattern [7, 8] and a double vortex [7, 9, 10]. Vortex structures have been observed [11, 12, 13] in low-temperature laboratory devices in which electron clouds are confined by dc electric and magnetic fields. Furthermore, large amplitude EDMs can parametrically excite electron zonal flows [14], which play a decisive role in electron 
confinement in nonuniform magnetized plasmas.

In this paper, we present the turbulent properties of nonlinearly interacting low-frequency (in comparison with the electron gyrofrequency) electron plasma waves (or EDMs) in a nonuniform magnetoplasma containing the electron density inhomogeneity. The manuscript is organized in the following fashion. In Sec. II, we discuss our 2D model equation for nonlinear EDMs and the possibility of frequency condensation due to the mode coupling process. Simulation results for a random initial turbulence state are described in Sec. III. In Sec. IV, we seek to understand the emergence of large-scale coherent structures by virtue of exact analytic solutions. The evolution of turbulent energy and the corresponding transport are discussed in Secs. VI and VII, respectively. The conclusions are contained in Sec. VIII.

\section{BASIC EQUATIONS}

Let us consider a strongly magnetized electron plasma in the presence of the equilibrium density gradient $\left(\partial n_{0} / \partial x\right)$ across $\hat{\mathbf{z}} B_{0}$, where $\hat{\mathbf{z}}$ is the unit vector along the $z$ direction in a Cartesian coordinate system and $B_{0}$ is the strength of the external magnetic field. The ions are supposed to form an immobile uniform neutralizing background. The perpendicular component of the electron fluid velocity in the presence of nonlinearly coupled low-frequency (in comparison with the electron gyrofrequency $\omega_{c e}=e B_{0} / m_{e} c$, where $e$ is the magnitude of the electron charge, $m_{e}$ is the electron mass and $c$ is the speed of light in vacuum) electric field $\mathbf{E}=-\nabla \phi$, where $\phi$ is the electrostatic potential, is

$$
\mathbf{u}_{e \perp} \approx \frac{c}{B_{0}} \hat{\mathbf{z}} \times \nabla \phi+\frac{c}{B_{0} \omega_{c e}}\left(\frac{d}{d t}+\nu_{e}-\mu_{e} \nabla_{\perp}^{2}\right) \nabla_{\perp} \phi,
$$

where $d / d t=(\partial / \partial t)+\left(c / B_{0}\right) \hat{\mathbf{z}} \times \nabla \phi, \mu_{e}$ is the electron collision frequency, and $\mu_{e}$ is the electron kinematic gyro-thermal-viscosity. The nonlinearity in (1) comes from the nonlinear electron polarization drift. The electron advection term $v_{e z} \partial / \partial z$ does not appear in $d / d t$ because of our 2D approximation.

Inserting (1) into the electron continuity equation and using the Poisson equation, we obtain the modified NSE

$$
\left[\frac{d}{d t}+\frac{\omega_{p e}^{2}}{\omega_{H}^{2}}\left(\mu_{e}-\mu_{e} \nabla_{\perp}^{2}\right)\right] \nabla_{\perp}^{2} \phi+\frac{\omega_{p e}^{2} \omega_{c e}}{\omega_{H}^{2}} K_{n} \frac{\partial \phi}{\partial y}=0,
$$

where $\omega_{p e}=\left(4 \pi n_{0} e^{2} / m_{e}\right)^{1 / 2}$ is the electron plasma frequency, $\omega_{H}=\left(\omega_{p e}^{2}+\omega_{c e}^{2}\right)^{1 / 2}$ is the 
upper-hybrid resonance frequency, and $K_{n}=-\partial n_{0} / \partial x$. In the absence of nonlinear interactions, Eq. (2) yields

$$
\omega=k_{y} u_{0}+\frac{\omega_{p e}^{2} \omega_{c e} k_{y} K_{n}}{\omega_{H}^{2} k_{\perp}^{2}}-i \frac{\omega_{p e}^{2}}{\omega_{H}^{2}} \Gamma,
$$

which is the frequency of the damped Doppler-shifted EDMs in the local approximation (viz. the wavelength being much smaller than the density gradient scalelength $K_{n}^{-1}$ ), and $\Gamma=\nu_{e}+\mu_{e} k_{\perp}^{2}$ is the damping rate. Here $\omega$ is the wave frequency, $\mathbf{k}=\mathbf{k}_{\perp} \equiv\left(k_{x}, k_{y}\right)$ is the wave vector, $u_{0}=\left(c / B_{0}\right) \partial \phi_{0} / \partial x \equiv-\left(c / B_{0}\right) E_{0}$ is the equilibrium electron drift in the presence of the dc electric field $E_{0}$, and $k_{\perp}^{2}=k_{x}^{2}+k_{y}^{2}$. In the absence of the dc electric field and dissipation, we observe from (3) that there exists frequency condensation at $k_{y} \gg k_{x}$. On the other hand, in a uniform plasma without $E_{0}$, we have the electron convective cell

mode [3],$\omega=-i\left(\omega_{p e}^{2} / \omega_{H}^{2}\right)\left(\nu_{e}+\mu_{e} k_{\perp}^{2}\right)$, which causes the cross-field electron transport due to random walk of the electrons in the electron convective cell electric field, even in a thermal equilibrium plasma [3].

Equation (2) describes the evolution of the electron fluid vorticity in 2D plane, e.g. in the $(x, y)$ plane. The kinematic electron-thermal gyroviscosity (say due to turbulent fluctuations) has been introduced to accommodate the damping of fluctuations at short scales.

To investigate the turbulence dynamics, we should have the knowledge of constant of motions of Eq. (2). The latter, without dissipation, admits two conserved quantities, namely the energy and enstrophy (squared vorticity)

$$
W=\int\left(\nabla_{\perp} \phi\right)^{2} d x d y, \quad \Omega=\int\left(\nabla_{\perp}^{2} \phi\right)^{2} d x d y,
$$

which show that the energy and mean squared vorticity are conserved ideally (or inviscidly) by $2 \mathrm{D}$ nonlinear interactions in a strongly magnetized electron plasma.

\section{SIMULATION RESULTS -FORMATION OF COHERENT STRUCTURES}

The modified NSE Eq. (2) has been integrated numerically with the help of a fully de-aliased pseudospectral scheme. Periodic boundary conditions are imposed along the $x$ and $y$-directions. The electrostatic potential is discretized in a Fourier space using $f(\mathbf{k}, t)=\sum_{\mathbf{k}} f(\mathbf{r}, t) \exp (-i \mathbf{k} \cdot \mathbf{r})$. All fluctuations in our simulations are initialized with 

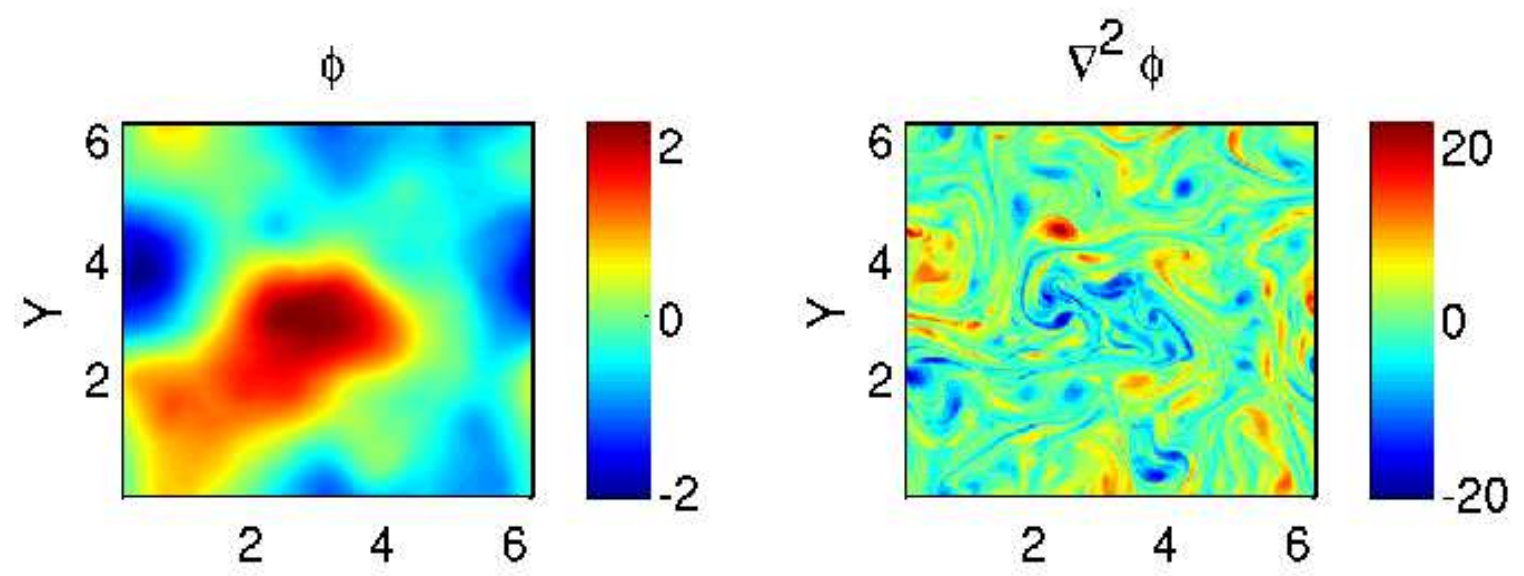

FIG. 1: Simulation results from a random initial state lead to the formation of large scale coherent mode in potential fluctuations owing to an inverse cascade process (see left fig). Figure on the right shows the small scale eddies that are formed essentially from the forward cascades of electron fluid vorticity at a similar time. This is called a dual cascade phenomenon, observed ubiquitously in many $2 \mathrm{D}$ turbulent systems. Here we have chosen $\Gamma / \omega_{c e}=0.01$ and $\beta=\left(\omega_{p e}^{2} / \omega_{H}^{2}\right) K_{n} L=2$, where $L$ is the box size.

a Gaussian random number generator to ensure that the Fourier components are all spatially uncorrelated and randomly phased. This ensures that the choice of initial state is highly isotropic, i.e. $k_{x} \approx k_{y}$ at $t=0$. Similarly, the boundary conditions (periodic in $x, y$ directions) do not impose any kind of anisotropy. Moreover, the results to be presented here are independent of the size of computational domains, number of Fourier modes, as well as the integration time steps.

The initially normalized energy spectrum, peaked at $k_{\min }$, is chosen to lie within the wavenumber interval $k_{\min }<k<k_{\max } / 2$. During the evolution of our simulations, the turbulence eventually evolves through vortex-merging in which like-signed smaller lengthscale fluctuations merge to form relatively large-scale fluctuations. The process continues until all merging has occurred to finally form the largest scale coherent vortex dominated by the minimum allowed $k$ in the simulation. The inertial range turbulent cascades in such a manner leads to the formation of large scale structures in potential fluctuations. By contrast, the vorticity structures break up and progressively form smaller eddies by means of a forward cascade processes. The large scale potential field nonetheless coexist with the 
small scale vorticity fluctuations and lead essentially to a dual cascade process. This is shown in Fig. (1) when the turbulence has reached its saturated state. The formation of large scale potential and small scale vorticity structures through nonlinear interactions can be understood in the context of a dual cascade phenomenon as the $2 \mathrm{D}$ electron plasma system admits two inviscid invariants. Under this process the potential cascades towards longer length-scales, while the fluid vorticity transfers spectral power towards shorter lengthscales. The randomly excited Fourier modes transfer the spectral energy in the inertial range by conserving the constants of motion (i.e. the inviscid invariants) in $k$ space. This leads to a statistically stationary inertial regime associated with the forward and inverse cascades. The dual cascades in the electron plasma wave turbulence is similar to the one that occurs in 2D hydrodynamic turbulence. The energy cascades towards smaller scales in the simulations is terminated essentially by a kinematic gyro-viscous damping. The latter is efficient at the smaller turbulent scales. The dual cascade processes reported here are further consistent with the number of inviscid constants (see Eq. (44) as admitted by the underlying system of Eq. (2).

Note that the presence of the density gradient convection caused by mean electric field does not quantitatively alter the characteristic nonlinear features of 2D electron plasma wave turbulence, as it exhibits a cascade property similar to that of the Charney-HasegawaMima equation. The evolving relaxation of the turbulent fluid is independent of spatial and temporal resolutions, as well as higher turbulent Reynolds numbers. The latter slow the rate of relaxation, while the qualitative physics remains more or less unaltered. Interestingly, we notice the formation of thin current sheet like structures in the evolution of the vorticity as shown in Fig. (11). Furthermore, the formation of the observed large scale structures in our simulations can be understood from an exact nonlinear analytic solution of Eq. (2). This is described below.

\section{ANALYTICAL SOLUTIONS FOR A DOUBLE VORTEX}

An analytic understanding of the emergence of coherent nonlinear structures observed in our fluid simulation can be achieved merely qualitatively by considering exact nonlinear solutions of the potential in Eq. (2). The underlying physical mechanism for the formation of coherent structures rests on the nonlinear interactions between different scalesize modes. In 
saturated states, the vector product nonlinearity overwhelms the dissipation, and a balance between the wave dispersion and nonlinearity gives rise to long-lived stable vortex structures that we observed in our simulations.

The two-dimensional traveling wave solutions of Eq. (21), without dissipation, in the moving frame can be obtained by supposing that $x=x, \xi=y-u t, t=t$, where $u$ being the speed of the vortex. This further transforms the fluctuating quantities into $\phi(x, y, t)=$ $\phi(x, \xi)$. The potential fluctuation obeys the localized boundary conditions $\phi \rightarrow 0$ as $y \rightarrow \infty$ for all $x$. The condition of localization associated with the coherent structures further enables us to use the periodic boundary conditions in computational domain of the nonlinear fluid simulations. The perturbed entities, or coherent structures, in the local region are far from the boundary and can translate freely. Hence no boundary effects are considered in our analysis. Boundary effects can nevertheless be important for certain processes, but this issue is well beyond the scope of the present work. Moreover, the $x-\xi$ plane in the simulations is orthogonal to the background magnetic field. On using the transformed co-ordinate systems, the electron vorticity Eq. (22) can be translated into a co-ordinate system that is moving along $\xi$-direction with the vortex speed. Following the previous treatment of the vortex theory [15, 16, 17, 18, 19, 20], we write (2) in the stationary frame

$$
D_{\xi}\left(\nabla_{\perp}^{2} \phi-\frac{u_{*}}{\left(u-u_{0}\right)} \phi\right)=0
$$

where $D_{\xi}=(\partial / \partial \xi)-\left[c /\left(u-u_{0}\right) B_{0}\right][(\partial \phi / \partial x)-(\partial \phi / \partial \xi) \partial / \partial x], \nabla_{\perp}^{2} \phi=\left(\partial^{2} \phi / \partial x^{2}\right)+\left(\partial^{2} \phi / \partial \xi^{2}\right.$, and $u_{*}=-\left(\omega_{p e}^{2} \omega_{c e} / \omega_{H}^{2}\right) K_{n}$. Equation (5) is satisfied by

$$
\nabla_{\perp}^{2}=C_{1} \phi+C_{2} x
$$

where $C_{1}$ and $C_{2}$ are constants, and $C_{1}+\left[\left(c /\left(u-u_{0}\right) B_{0}\right] C_{2}=u_{*} /\left(u-u_{0}\right)=\alpha^{2}\right.$. For $\alpha^{2}>0$, Eq. (6) admits a double vortex [15]. The profiles of the latter in the outer $(r>R$, where $r^{2}=x^{2}+\xi^{2}$ and $R$ is the vortex radius) and inner $(r<R$; centered at the point $x=\xi=0)$ regions are, respectively,

$$
\phi(r, \theta)=\phi_{0} K_{1}(\alpha r) \cos \theta
$$

and

$$
\phi(r, \theta)=\left[\phi_{i} J_{1}(\gamma r)+D_{i} r / \gamma^{2}\right] \cos \theta,
$$




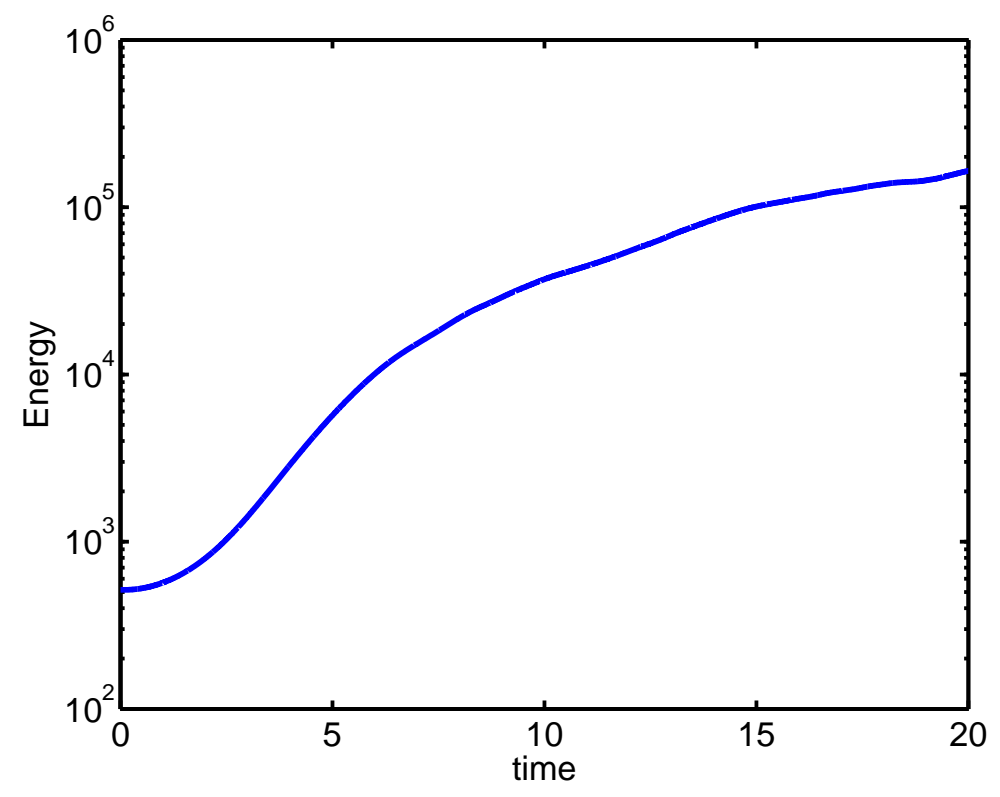

FIG. 2: Evolution of the total energy in the simulation.

where $\cos \theta=\xi / r, \phi_{0}=D_{i} R /\left(\alpha^{2}+\gamma^{2}\right) K_{1}(\alpha R), \phi_{i}=-\alpha^{2} D_{i} R / \gamma^{2}\left(\alpha^{2}+\gamma^{2}\right) J_{1}(\alpha R), D_{i}=$ $\left(\alpha^{2}+\gamma^{2}\right)\left(u-u_{0}\right) B_{0} / c$, and the constant $\gamma$ has to be determined from the transcendental equation

$$
\frac{K_{2}(\alpha R)}{\alpha K_{1}(\alpha R)}=-\frac{J_{2}(\gamma R)}{\gamma J_{1}(\gamma R)}
$$

Here $J_{1}\left(J_{2}\right)$ and $K_{1}\left(K_{2}\right)$ are the Bessel and modified Bessel functions of the first (second) order, respectively. It turns out that the presence of the density gradient is essential for the formation of a double vortex.

\section{EVOLUTION OF TURBULENT ENERGY AND SPECTRUM}

The evolution of the turbulent energy associated with the potential and vorticity fields is shown in Fig. (21) where the linear and nonlinear phases of the evolution are clearly marked by the curve. The nonlinear Eq. (2), in the absence of dissipation, conserves the total energy. In the presence of dissipation, small scales are dissipated. Since the 2D electron plasma system is dominated by the smaller $k$ 's, the large scale turbulent eddies tend to grow and contain most of the turbulent energy. The volume integrated energy therefore grows in time until the steady state is reached. The evolution of the energy is 


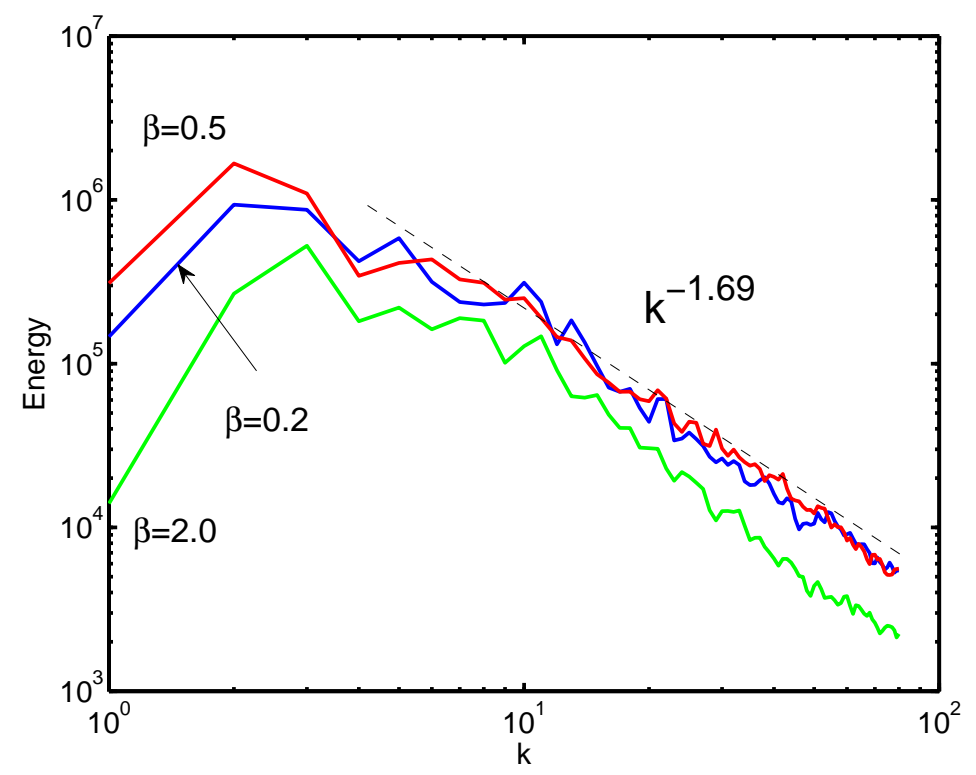

FIG. 3: The 2D electron plasma turbulence in the presence of the density gradient (maintained by an external electric field) exhibits a turbulent spectrum close to the Kolmogorov-like $k^{-5 / 3}$ scaling over the inertial range. The effect of a variation of the mean electric field is also shown in the turbulent spectrum.

shown in Fig. (2). This is further consistent with the formation of large-scale coherent convective cells/flows in which the energy associated with turbulence must evolve in order to relax the turbulence into a well organized coherent structure. After nonlinear interactions are saturated, the energy in the turbulence does not grow and remains nearly unchanged throughout the simulations. Correspondingly, the energy transfer rate shows a significant growth during the linear and initial nonlinear phases (not shown in Fig. (2)). However, when nonlinear interactions saturate, the nonlinear transfer of the energy in the spectral space amongst various turbulent modes becomes inefficient and the energy transfer per unit time tends to become negligibly small.

The power spectrum associated with the electron plasma turbulence exhibits a spectral slope close to a Kolmogorov-like scaling $k^{-5 / 3}$, as shown in Fig. (3). This is indicative of the eddy interaction being the dominant process in the spectral cascades of the inertial range turbulent energy despite the presence of the convection of the density gradient by the mean electric field, and is consistent with the Kolmogorov-like phenomenology. 


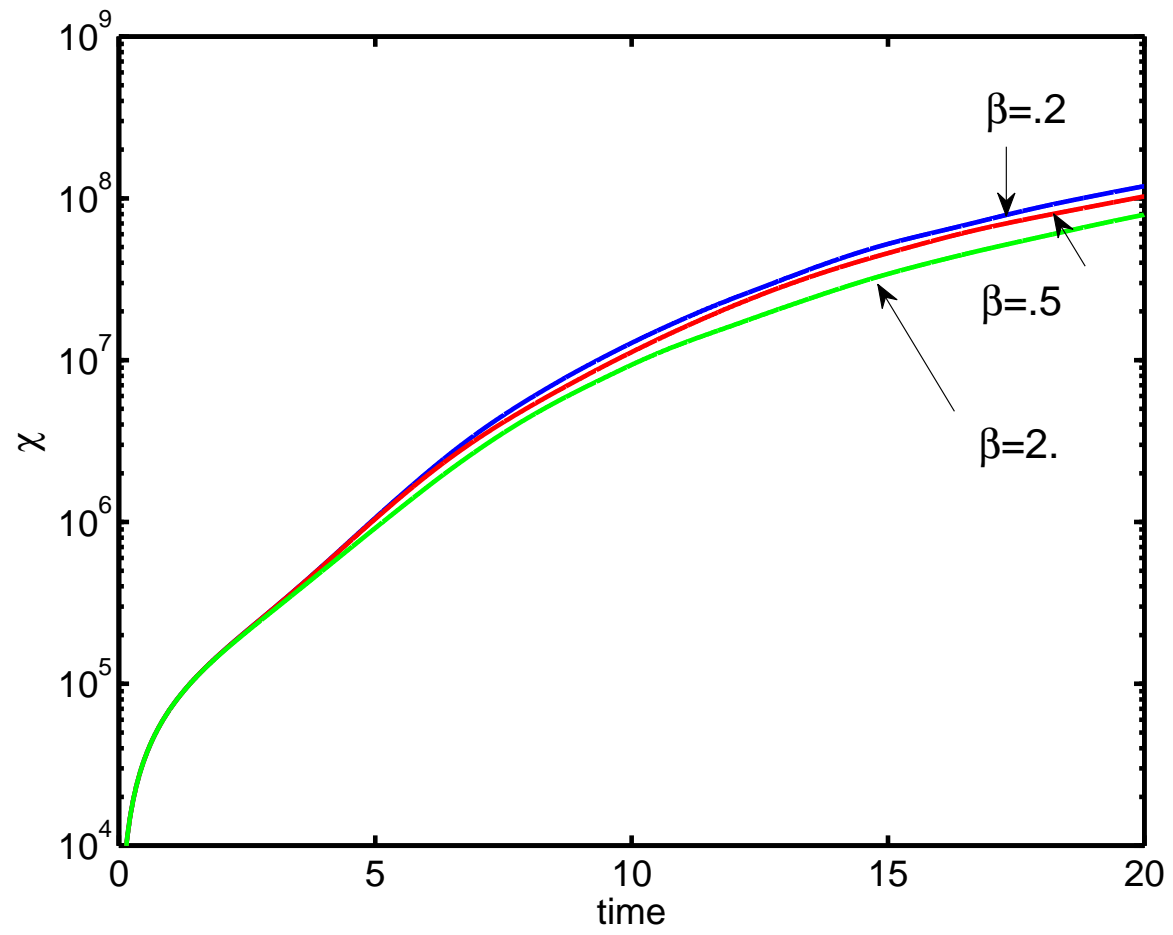

FIG. 4: Evolution of the effective diffusion coefficient is studied in the presence of the density gradient in a nonuniform $2 \mathrm{D}$ electron magnetoplasma. Increasing strength of the inhomogeneity leads to a reduction in the net electron transport across the magnetic field lines. This result is also consistent with the experimental observations.

\section{TURBULENCE TRANSPORT}

The cross-field electron transport is triggered primarily by the mutual collisions of the electrons in the presence of an ensemble of coherent structures. Predominantly, it is the successive collisions amongst the electrons that can potentially lead to a net outward drift across the external magnetic field. However, the presence of an external orthogonal electric field is believed to improve the stability of $2 \mathrm{D}$ electron plasma. Motivated by this observation, we investigate here the transport of electrons that is influenced by turbulent evolution of the potential and vorticity in 2D electron plasma. We follow the evolution of the effective diffusion by increasing the magnitude of externally imposed mean electric field. An effective electron diffusion coefficient can be calculated from $D_{\text {eff }}=\int_{0}^{\infty}\left\langle\mathbf{V}_{\perp}(\mathbf{r}, t) \cdot \mathbf{V}_{\perp}\left(\mathbf{r}, t+t^{\prime}\right)\right\rangle d t^{\prime}$ where the angular bracket represents spatial averages. The perpendicular component of the electron fluid velocities in $2 \mathrm{D}$ electron plasma is $\mathbf{V}_{\perp} \approx \hat{\mathbf{z}} \times \nabla \phi$. Since the 2D EDM tur- 
bulence is confined in a plane orthogonal to the ambient magnetic field in $\hat{\mathbf{z}}$ direction, the effective cross-field diffusion coefficient, $D_{\text {eff }}$, essentially describes the diffusion processes associated with the transverse motion of the electrons in $x-y$ plane. We compute $D_{\text {eff }}$ in our simulations to measure the turbulent transport that is associated with the intermittent electron plasma wave turbulence. It is observed that the effective cross-field transport is lower, when the field perturbations are Gaussian. On the other hand, the cross-field diffusion increases rapidly with the eventual formation of longer length-scale structures. This is shown in Fig. (4), which exhibits a dependence of $D_{\text {eff }}$ with time. Thus, an enhanced crossfield transport level results primarily due to an emergence of large-scale coherent structures in a nonuniform magnetized plasma. The enhanced cross-field diffusion coefficient observed in our simulations is, therefore, consistent with the generation of large-scale flows.

The most remarkable point to emerge from our simulations is the reduction of the electron transport across the confining magnetic field when the strength of the applied or external electric field is increased. We have reported three different cases for the evolution of the diffusion coefficient corresponding to $\beta=0.2,0.5$ and 2.0 in Fig. (4). It is clear from Fig. (4) that the effective transport associated with the electron plasma waves is much better (see the lower curve in Fig. (4) for $\beta=2.0$ ) for a higher strength of the applied or external electric field. Our simulation results are further consistent with the experimental observation of Ref. [2].

\section{DISCUSSION}

In this paper, we have studied the turbulent properties of nonlinearly interacting lowfrequency EDMs in a nonuniform magnetoplasma by using computer simulations. Specifically, we have numerically investigated the modified Navier-Stokes equation (2) and have studied spectral cascades and cross-field electron transport produced by nonlinearly saturated turbulent modes in such a plasma system. It is found that self-organized coherent structures emerge due to nonlinear interactions between different scale sizes $2 \mathrm{D}$ fluctuations. The final state is thus intermittent and exhibits a dual cascade in which a forward cascade of the electron vorticity coexists with an inverse cascade of the potential fluctuation. The inertial power spectrum is close to the Kolmogorov-like $5 / 3$ scaling, whereas the transport is dominated by large scale vortical structures. We find that the stability of the electron 
plasma column confined by a straight magnetic field can be improved by virtue of an external electric field maintaining the density inhomogeneity, a result that is also consistent with experimental observations [2].

[1] R. C. Davidson, Physics of Nonneutral Plasmas (Addison Wesley, Redwood City, 1990).

[2] F. Anderegg, E. M. Hollmann, and C. F. Driscoll, Phys. Rev. Lett. 81, 4875 (1998).

[3] H. Okuda and J. M. Dawson, Phys. Fluids 16, 480 (1973); P. K. Shukla, M. Y. Yu, H. U. Rahman, and K. H. Spatschek, Phys. Rep. 105, 227 (1984).

[4] D. A. Shechter and D. E. Dubin, Phys. Rev. Lett. 83, 2191 (1999).

[5] Y. Kiwamoto, K. Ito, A. Sanpei, and A. Mohri, Phys. Rev. Lett. 85, 3173 (2000).

[6] Y. Kiwamoto, N. Nashizume, Y. Soga, J. Aoki, and Y. Kawai, Phys. Rev. Lett. 99, 115002 (2007).

[7] P. K. Shukla, in A Variety of Plasmas, edited by A. Sen and P. K. Kaw (Indian Academy of Sciences, Banglore, India, 1991), pp. 297-314.

[8] S. N. Antani, A. Sen, and S. Roy Choudhury, Phys. Scr. 42, 581 (1990).

[9] P. K. Shukla, J. Srinivas, G. Murtaza, and H. Saleem, Phys. Plasmas 1, 3505 (1994).

[10] J. O. Hall and P. K. Shukla, Phys. Plasmas 12, 12230 (2005).

[11] N. A. Kervalishvili, Phys. Lett. A 157, 391 (1991).

[12] G. Rosenthal, A. Y. Wong, and S. Bauer, Phys. Lett. A 170, 443 (1992).

[13] A. J. Peurrung and J. Fajans, Phys. Fluids A 5, 493 (1993); D. Durkin and J. Fajans, Phys. Rev. Lett. 85, 4052 (2000).

[14] P. K. Shukla and L. Stenflo, Phys. Lett. A 352, 431 (2006).

[15] V. D. Larichev and G. M. Reznik, Oceanology 16, 547 (1976).

[16] J. D. Meiss, W. Horton, Phys. Fluids 4, 990 (1983).

[17] V. I. Petviashvili and O. A. Pokhotelov, Solitary Waves in Plasmas and in The Atmosphere (Gordon Breach, London, 1992).

[18] L. Stenflo, Phys. Fluids 30, 3297 (1987).

[19] W. Horton and A. Hasegawa, Chaos 4, 227 (1994).

[20] P. K. Shukla, G. T. Birk, and R. Bingham, Geophys. Res. Lett. 22, 671 (1995). 